\title{
Adverse Drug Reaction of Antifungals in the Management of Black Fungus: A Tertiary Care Centre Experience
}

\author{
Kavita Sachdeva $^{1}$ - Tinnu Anna Saji ${ }^{1}$ (D)
}

Received: 28 June 2021/Accepted: 21 August 2021/Published online: 1 September 2021

(C) Association of Otolaryngologists of India 2021

\begin{abstract}
In India, COVID-19 has led to a surge in cases of a potentially fatal fungal infection called mucormycosis, popularly known as "black fungus." Intravenous amphotericin B is the only available drug for salvage therapy. Efforts to improve its therapeutic efficacy and decrease its nephrotoxicity have focussed on the reformulation of AmB in three new lipid formulations such amphotericin B lipid complex (Abelcet), amphotericin B colloidal dispersion (Amphotec), and liposomal amphotericin B (AmBisome). The aim of this study is (1) to evaluate the adverse drug reaction of various formulations of amphotericin B used for the treatment of rhinooculocerebralmucormycosis in Indian population. (2) to evaluate the adverse drug reaction of injectable form of posaconazole. This prospective observational study was done on a random sample of 110 patients who got admitted for the management of rhinooculocerebral mucormycosis in a tertiary care centre of middle india... The patients were assessed for the adverse reactions following the administration of various antifungal medication and the findings were analysed. All the 110 patients had received two forms of Amphotericin B (liposomal Amphotericin B and Amphotericin B lipid complex) and Posaconazole injection. 60 patients had received all three forms of Amphotericin B. Out of the 110 patients who received Liposomal amphotericin B, only 2 patients developed adverse drug reaction while in 110 patients who received Amphotericin B Lipid complex, 7 patients had adverse drug effects. Lyophilised amphotericin B had been
\end{abstract}

Tinnu Anna Saji

anusaji1989@gmail.com

Kavita Sachdeva

drksachdeva@yahoo.co.in

1 Department of ENT, NSCB MCH, Jabalpur, India administered to 60 patients in which 51 patients developed adverse drug reaction and in them one patient went to congestive cardiac failure. Injection posaconazole had been administered to 110 patients in which 72 patients developed drug reaction. In spite of its proven track record of Amphotericin B, its well-known side effects and toxicity will sometimes require discontinuation of therapy despite a life-threatening systemic fungal infection. Lipid formulations of $\mathrm{AmB}$ are better tolerated than $\mathrm{AmB}$ deoxycholate but infusional drug reactions have been reported in lipid formulation too. So improved strategies for the management of infusion related adverse events are required.

Keyword Black fungus - Adverse drug reaction . Antifungals

\section{Introduction}

The rapid global spread of the Coronavirus disease led to the declaration of COVID-19 as a pandemic on March 11, 2020 (Organization, 2020). As India continues to achieve stability over the existing situation, another imminent threat has emerged as a challenge to India in the form of coronavirus disease-associated mucormycosis. Mucormycosis, caused by a group of moulds called mucormycetes, is a rare but potentially fatal infection if inadequately treated. Often referred to as the so-called black fungus, the incidence of mucormycosis has risen more rapidly during the second wave compared with the first wave of COVID-19. The fungus blocks blood flow, which kills infected tissue, and it is this dead, or necrotic, tissue that causes Rhinoorbital-cerebral the characteristic black discoloration of people's skin, rather than the fungus itself. Infection usually originates from the paranasal sinuses, with bone 
destruction and subsequent invasion of the orbit, and braincausing sinoorbital and/or rhinocerebral infections, respectively. The COVID-19 causes favourable alteration in the internal milieu of the host for the fungus and the medical treatment given, unwittingly also abets fungal growth. Uncontrolled Diabetics and Medications like steroids increase blood sugar, serum acidosis causes an increase in the serum iron which is very important for the fungus to grow. Long-term ventilation reduces immunity and there are speculations of the fungus being transmitted by the humidifier water being given along with oxygen. Broad-spectrum antibiotics not only wipe out the potentially pathogenic bacteria but also the protective commensals. Amphotericin B, which destroys the cell wall of the fungus, is the first-line medical treatment for mucormycosis and should be initiated as soon as the diagnosis is suspected. Efforts to improve its therapeutic efficacy and decrease its nephrotoxicity have focused on the reformulation of $\mathrm{AmB}$ in various lipid delivery vehicles like amphotericin B lipid complex(Abelcet), Amphotericin B colloidal dispersion (Amphotec), and liposomal amphotericin B (AmBisome).

\section{Methods}

\section{Study Area and Target Population}

This prospective observational study was done on 110 patients who got admitted to rhinoorbitocerebral mucormycosis ward of a tertiary care centre in middle india receiving antifungal medication between May 2021 to June 2021

\section{Study Design}

This is a prospective observational study

\section{Inclusion Criteria}

- Patients receiving antifungal medication

- Patients giving consent for the study.

\section{Exclusion Criteria}

- Patients who refused antifungal medication

- Patients with known allergy to antifungal medication

- Patients with derranged renal parameters.

\section{Data Collection}

- Detailed history regarding the Covid infection,symptoms,hospitilisation and medication history, antifungal received, symptoms developed following medication,physical examination and all the necessary investigations were done for the patients.

- Amphotericin B administered to the patient.Dose calculated as per the weight of patient, the extent of disease.renal parameters of the patient.Injection and tablet posaconazole were given to the patient who are intolerable to Amphotericin B, compromised renal function,electrolyte imbalance and in non availability of Amphotericin B.

- The data collected was entered in Microsoft excel and analysed.Analyzed data was presented in the form of frequency and percentage shown by pie and bar chart.

\section{Ethical Approval}

Ethical approval for this was obtained from the institutional ethics committee.

\section{Ethical Issues}

None

\section{Statistical Analysis}

The data collected was entered in Microsoft excel and analysed. Analyzed data was presented in the form of frequency and percentage shown by pie and bar chart.

\section{Results}

Out of the 110 patients who received liposomal Amphotericin $\boldsymbol{B}$ injection, 2 patients developed adverse drug reaction (Fig. 1). Both of them were below 50 years of age and developed chills, and rigor within 15 minutes of drug administration.

110 patients received Amphotericin B Lipid complex, in which 7 patients developed adverse drug reaction (Fig. 2). 3 patients were between 61 and 70years, 2 between 31 and 40years, 2 patients between 40 and 60years of age (Fig. 3).The adverse drug reaction observed were chills and rigor(38\%),fever (23\%), thrombophlebitis(23\%), infraorbital swelling(8\%), itching(8\%) (Fig. 4) 


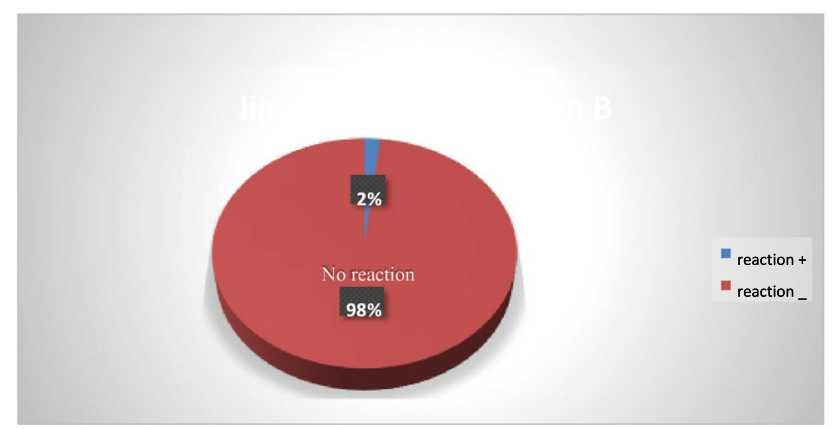

Fig. 1 Incidence of adverse drug reaction with liposomal Amphotericin $\mathrm{B}$

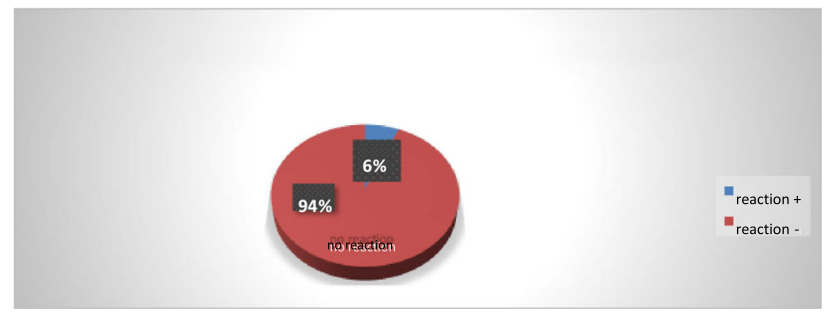

Fig. 2 Incidence of adverse drug reaction with Amphotericin b lipid complex

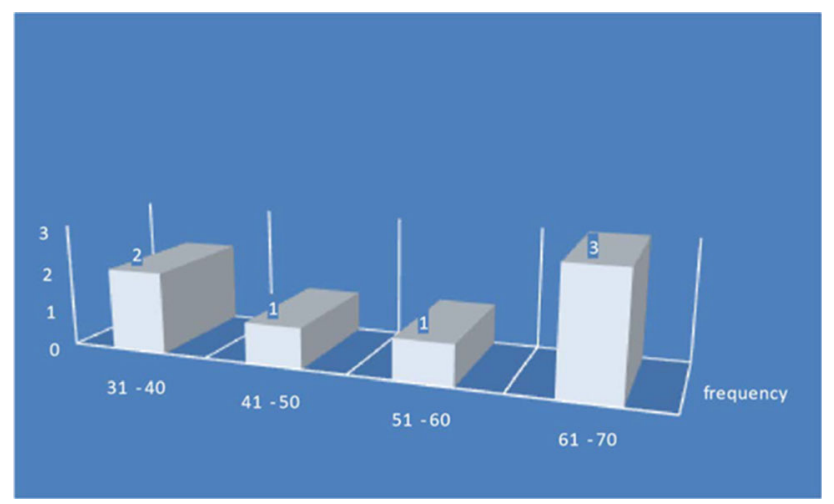

Fig. 3 Age wise incidence of adverse drug reaction with Amphotericin B lipid complex

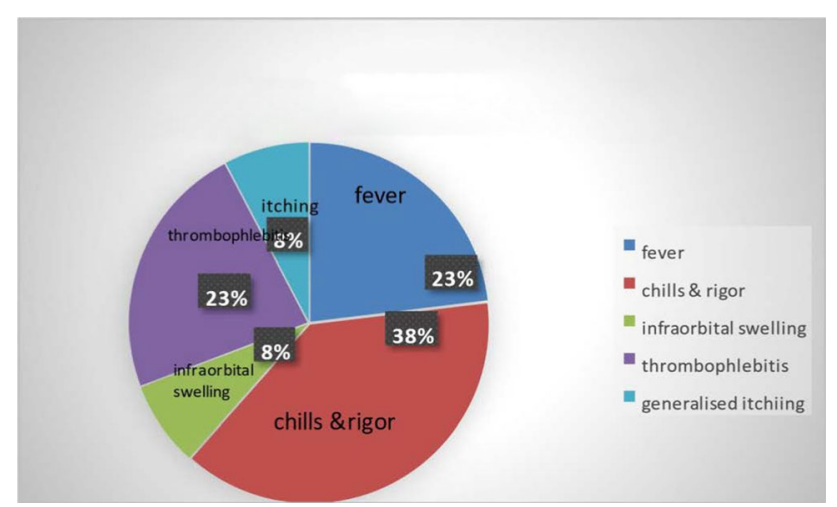

Fig. 4 Adverse drug reaction with Amphotericin B lipid complex
Out of the total 60 patients who received lyophilised Amphotericin B injection,51 patients (85\%) got adverse drug reaction (Fig. 5) in which 19 were between age of 41-50,15 patients between 51 and 60,5 patients less than 40 , rest 8 patients above 60 years of age (Fig. 6). 16 patients developed reaction in 10 minutes, 11 patients (15 minutes), 8(20minutes), 6 ( 5 minutes), 1 (25 minutes), and 5 (30 minutes) (Fig. 7). The adverse drug reactions were shivering (42patients), fever (33patients), saturation drop and breathlessness (8 patients), nausea, vomiting (6patients) and headache (6 patients), bodyache and weakness (5 patients), chestpain (4 patients), rashes and dryness of mouth (1 patient) (Fig. 8)

110 patients who received posaconazole injection, 72 developed adverse drug reaction (Fig. 9) in which 25 people were in the age group 51-60years, 13(61-70years), 12(31-40years), 12(41-50years),3(7180years) (Fig. 10). The adverse drug reactions were thrombophlebitis(54), fever(29), chills (250, headache(4), vomiting(3), sweating(1) (Fig. 11).

\section{Discussion}

The efficacy and safety (i.e., therapeutic index) of any liposomal AMB product is primarily determined by the rate and extent of $\mathrm{AMB}$ release from the liposomes upon administration. First, the small size of these liposomes $(<100 \mathrm{~nm})$ results in prolonged circulation allowing distribution into many different organs [1]. Second, its composition ensures that AMB remains associated with the liposome bilayer until it comes into contact with a fungus, minimising the adverse effects of AMB on host tissues.

In the present study out of the 110 patients who received Liposomal Amphotericin B, 2 patients developed adverse drug reaction. The reaction observed were chills and rigor which developed within 15 minutes of drug administration

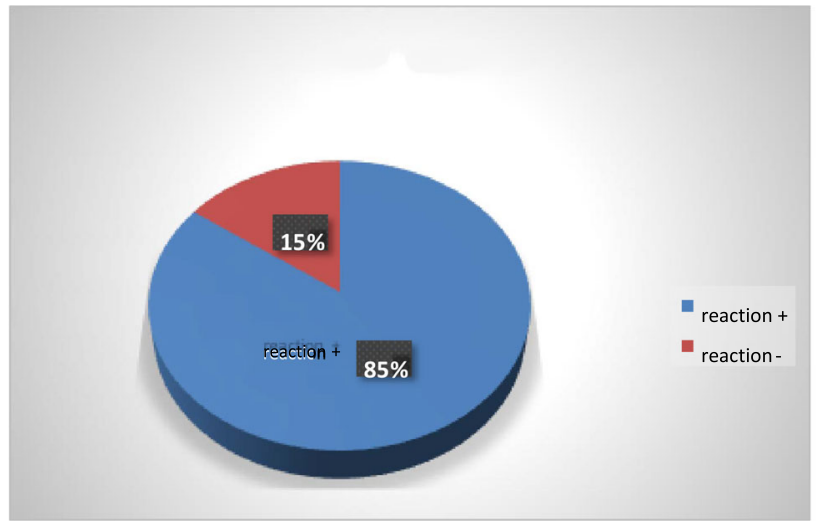

Fig. 5 Incidence of adverse drug reaction with lyophised Amphotericin B 


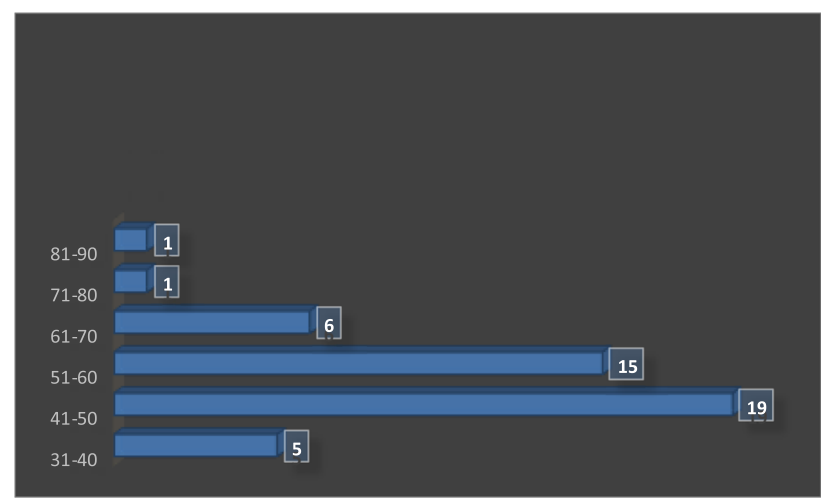

Fig. 6 Age wise incidence of adverse drug reaction with lyophilised $B$ injection

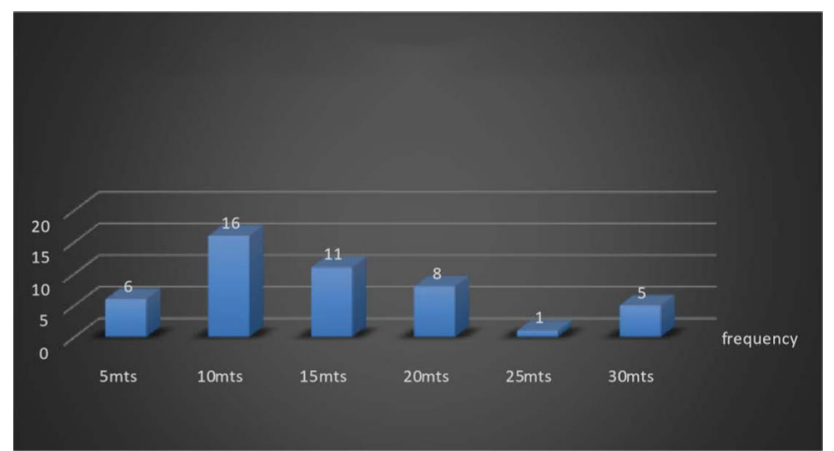

Fig. 7 Time of onset of adverse drug reaction with lyophilised amphotericin injection

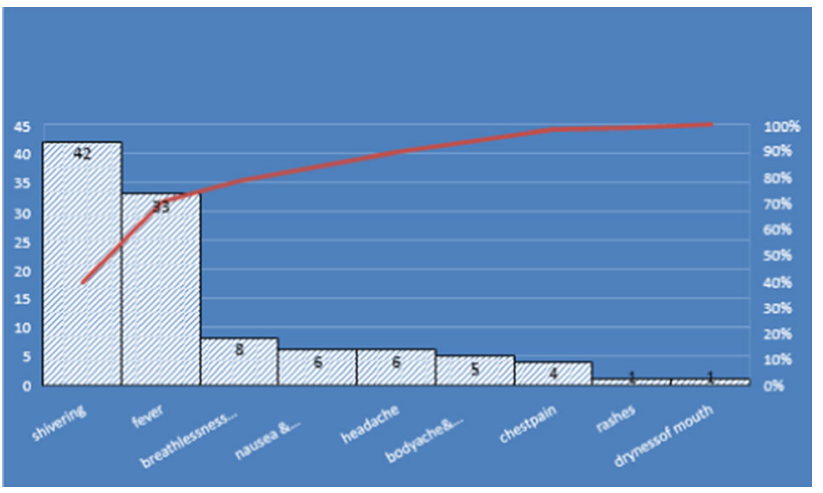

Fig. 8 Adverse drug reaction with lyophilised Amphotericin injection

and got subsided with the administration of avil and decadron. Both had received Liposomal Amphotericin B for the first time. On subsequent injections of liposomal Amphotericin B, they had no reaction. One of the above patient had developed reaction to injection posaconazole too. There were no underlying risk factors or drug interactions in this patients.

A more recent multicenter study on acute infusion-related reactions to liposomal $\mathrm{AmB}$ reported that acute

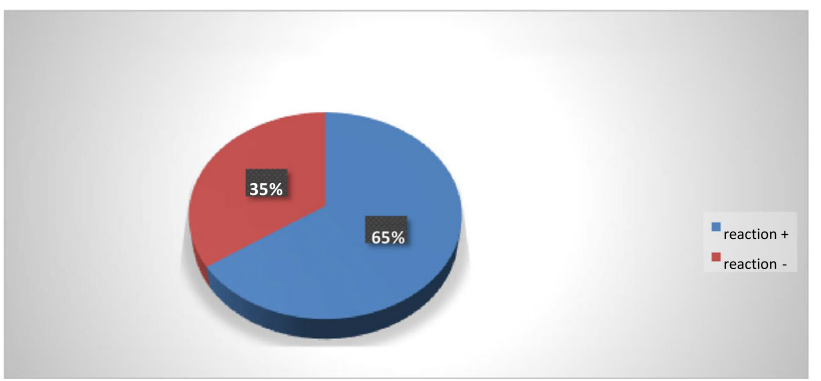

Fig. 9 Incidence of adverse drug reaction with posaconazole injection

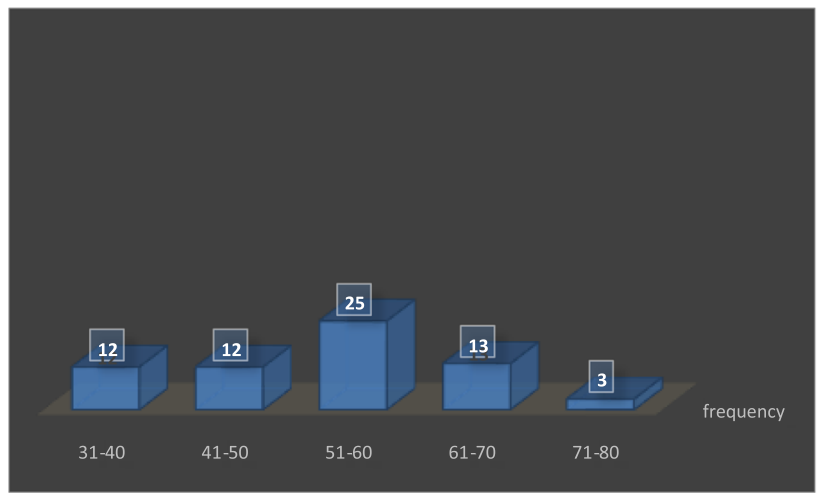

Fig. 10 Age wise incidence of adverse drug reaction with posaconazole injection

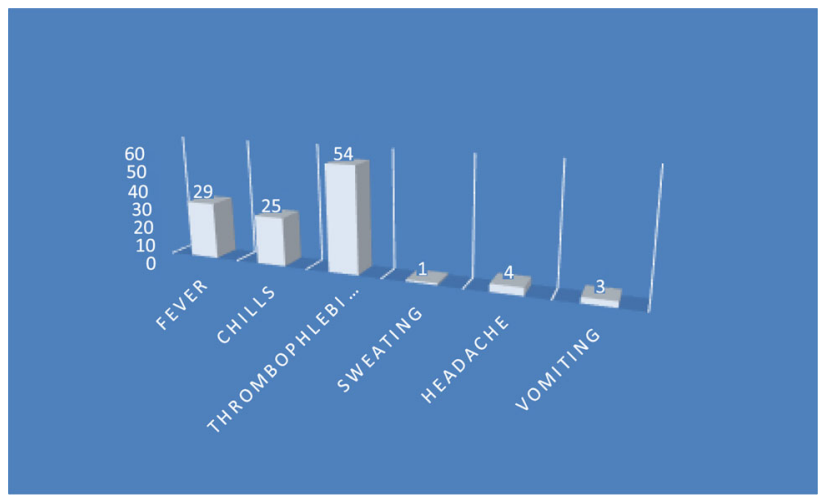

Fig. 11 Adverse drug reaction with injection posaconazole

adverse effects occurred alone or in combination within 1 of 3 symptom complexes: (1) chest pain, dyspnea, and hypoxia; (2) severe abdomen, flank, or leg pain; and (3) flushing and urticaria. Most adverse reactions (86\%) occurred within the first $5 \mathrm{~min}$ of infusion. All patients experienced rapid resolution of symptoms after IV diphenhydramine [2]. A more dangerous side effect of rapid IV infusion is hyperkalemia secondary to shift of administration. The analysis demonstrated an overall frequency of infusion-related reactions of $20 \%$ [3] with the potential for the development of fatal cardiac potassium 
from the intracellular compartment arrhythmias [4]. The reaction is not a dose-dependent phenomenon. As the clinical syndrome of infusion reaction is more similar to other liposome-associated drugs (such as liposomal doxorubicin) than to $\mathrm{DAmB}$, the reaction may be to the liposome rather than the active drug. The mechanism remains unclear but is postulated to be complement mediated [5]. The recommended dose of liposomal Amphotericin B for Rhinooculocerebral mucormycosis is $5-10 \mathrm{mg} / \mathrm{kg} / \mathrm{day}$ for 14-21 days depending on severity of infection.

ABLC is a lipid complex consisting of AmB and the lipids dimyristoylphospatidylcholine and dimyristoylphosphatidylglycerol in a 1:1 molar ratio [6] $\mathrm{t}$ is believed that phospholipases released by vascular smooth muscle as well as those derived from macrophages and released by infecting fungi, break down the lipids complexed to amphotericin $\mathrm{B}$, thus releasing drug at the infection site. This may reduce nephrotoxicity [7]. Out of the 110 patients who received Amphotericin B lipid complex, 7 patients developed drug reaction.All the patients received ABLC for the first time. All the 7 patients had received Liposomal Amphotericin B priorly without any reaction. No specific age predilection had been noticed. Chills and rigor were observed in 5 patients within 15 minutes of administration and got subsided with antihistamine injection in 30 minutes. 3 patients developed fever which persist for next 12 hours. Thrombophlebitis at the injection site developed in 3 patients and infraorbital swelling in 1 patient which last for 1 week. Generalised body itching in 1 patient for 48 hours. Administration of amphotericin B lipid complex (ABLC) may be associated with infusion-related reactions, such as fever, rigors, and chills. Premedication with hydrocortisone may reduce the incidence of these reactions.In a prospective 18-month study, patients with cancers were given intravenous hydrocortisone $100 \mathrm{mg}$ 15-30 minutes before each infusion of ABLC (275 cycles; mean dose per cycle $931 \mathrm{mg}$ ) There were 44 infusion-related reactions (16\%), most of which followed the first infusion of a cycle $(15 \%$; subsequent infusions $2.9 \%$ ). The most common reactions were rigors (15\%) and fever (13). There was no significant difference in the rates or types of reactions between ABLCnaïve and previously treated patients. [8]

ABLC is available as a $100 \mathrm{mg} / 20 \mathrm{~mL}$ suspension in a single use vial.The recommended dose is $5 \mathrm{mg} / \mathrm{kg}$ body weight given as a single infusion over 2 hour.

Out of the 110 patients, 60 patients were initiated with lyophilised Amphotericin B, in which 51 developed severe adverse drug reaction which necessitated the sudden stoppage of drug. Majority of the population developed reaction (33) within 10 to 15 minutes and even 5 patients after 30 minutes of drug administration. The commonest symtoms were shivering(42) and fever(33) followed by breathlessness and saturation $\operatorname{drop}(8)$, nausea, vomiting, headache(6), weakness and bodyache(5), chestpain(4), rashes and dryness of mouth(1). Shivering got subsided in 30 minutes with the administration of antihistamine and decadron. Fever persisted for nearly 12 hours. Out of the 8 patients who developed breathlessness and saturation drop, 5 patients needed oxygen support at the rate of 3-5 litres for about 24 hours, while the rest 3 required about 5-8 litres of oxygen for about 48 hours. Nausea and vomiting persisted for nearly 48 hours and were administered with antiemetics.weakness and bodyache for nearly 3 days. 4 patients developed chestpain,ECG was normal in 3 of them, got relieved with symptomatic management and antianxiety drugs. But 1 of them had pre-existing cardiac disease with ejection fraction $35 \%$ went for congestive cardia failure. Antifungal might have precipitated his preexisting cardiac illness. Rashes persisted for nearly 72 hours. The recommended dosage of Amphotericin B is $1-1.5 \mathrm{mg} / \mathrm{kg} /$ day for $14-21$ days depending on severity of infection.

Amphotericin B exerts its effects by binding to specific chemical structures (sterols) of the fungal cellular membrane, increasing cellular permeability and promoting the loss of potassium and the leaking of other cell substances and subsequent cell death [9]. Each vial of Lyophilised Amphotericin B(affytericinB) contains a sterile, nonpyrogeni, lyophilised powder providing $50 \mathrm{mg}$ amphotericin B and sodium deoxycholate with sodium phosphate as a buffer, Crystalline Amphotericin B is insoluble in water,therefore antibiotic is solubilised by addition of sodium deoxycholate to form a mixture which provides a colloidal dispersion for intravenous infusion following reconstitution. It is reconstituted only withsterile water without preservatives (not bacteriostatic water containing benzyl alcohol, sodium chloride or other electrolyte solutions due to $\mathrm{AmB}$ precipitation/aggregation).The solution may then be further diluted with $5 \%$ dextrose, $10 \%$ dextrose, or $20 \%$ dextrose injection for IV infusion. A final concentration not exceeding $0.5 \mathrm{mg} / \mathrm{ml}$ in $5 \%$ or $10 \%$ dextrose injection may be administered through a central catheter via an-line filter ( $>1 \mathrm{~lm}$ mean pore diameter); however, ifperipheral administration is necessary, concentrations should not exceed $0.1 \mathrm{mg} / \mathrm{ml}$ in $5 \%$ dextrose injection [10].

Acute reactions such as fever with or without rigors, hypotension and, rarely, anaphylaxis are possible. These are usually seen within 90 min of administration and subside by 3 to $4 \mathrm{~h}$. Tolerance to amphotericin B therapy develops over time. Therefore, weekly reevaluation of the need for premedication is necessary. Premedication should consist of acetaminophen or hydrocortisone $25 \mathrm{mg}$ i.v., at $30 \mathrm{~min}$ prior to initiating $\mathrm{AmB}$ infusion, if the patient experiences severe rigors with previous infusion.Meperidine $25 \mathrm{mg}$ i.v. every $15 \mathrm{~min}$ up to $100 \mathrm{mg}$ over 
$1 \mathrm{~h}$ may be considered for the treatment of rigors. $1 \mathrm{~L}$ of $0.9 \%$ sodium chloride injection or $5 \%$ dextrose with $0.9 \%$ sodium chloride injection may be administered before or after amphotericin infusion to decrease some of the nephrotoxicity.

Doubling the dosage of amphotericin B and administering it every other day may diminish nausea. If azotemia occurs, dose of amphotericin B may be reduced or stopped based on renal function, assessment of risks versus benefits of continued therapy.

Posaconazole (Noxafil ${ }^{\circledR}$ ) is a systemic triazole antifungal drug derived from itraconazole and exerts the same antifungal mechanism of action as other azole derivatives [11]. Three formulations are currently available, namely an oral suspension $(40 \mathrm{mg} / \mathrm{mL})$, a delayed-release tablet (100 $\mathrm{mg}$ ), and an intravenous formulation $(18 \mathrm{mg} / \mathrm{mL})$. The posaconazole oral suspension and delayed-release tablet are approved for patients aged 13 years and older (USA) or adults aged18 years and older (Europe), while the intravenous formulation is licensed only for patients aged 18 years and older.

The posaconazole suspension is indicated to be administered as $200 \mathrm{mg}$ four times daily (TID) or $400 \mathrm{mg}$ twice daily in mucormycosis. The delayed-release tablet and intravenous formulation areindicated to be given as a loading dose at $300 \mathrm{mg}$ BID on the first day and a maintenance dose at $300 \mathrm{mg}$ once daily thereafter.

In the present study posaconazole injection had been administered to 110 patients in which adverse drug reaction developed in 72 patients.majority of the patients(25) in the age group of 50-60 years. The thrombophlebitis developed in 54 patients which persisted for nearly 1 week. The other common symptoms were fever and chills followed by headache and vomiting. Posaconazole injection should always be given through central line.In the present study it had been administered via peripheral line which might be the cause for the mass reaction.

The most frequently reported adverse events (AEs) during posaconazole treatment in other studies included gastrointestinal disorders-such as diarrhea, nausea, vomiting - and also hypokalemia, pyrexia, which are manageable from a clinical perspective [12-14].The other severe toxicity is hepatoxixcity and nephrotoxicity.

The long term adverse effect of Amphotericin B observed in the current study is abdominal distension, hypokalaemia and vomiting.

\section{Conclusions}

Patients with Covid associated mucormycosis should be managed with surgical debridement and antifungals. Amphotericin B is the only salvage therapy for rhinoorbitocerebralmucormycosis In spite of its proven track record of Amphotericin B, its well-known side effects and toxicity will sometimes require discontinuation of therapy despite a life-threatening systemic fungal infection,The two types of formulations Liposomal/lipid complexAMB and Amphotericin B deoxycholate are similar in efficancy but the latter needs to be monitored for electrolyte imbalance and nephrotoxicity.The adverse drug reactions have been observed in all formulation but least with lipid complex. Liposomal Amphotericin B remains the gold standard. Second choiceof drug is posaconazole suspension/sustained release tablet/injection.Injection posaconazole which should be always administered via central line. It is preferred in case of renal dysfunction,uncontrolled infusion reaction,electrolyte imbalance or unavailability of amphotericin B. Proper premedication,slow infusion of drug over 6-8 hours, pre and post hydration with $500 \mathrm{ml}$ Normal saline, close monitoring of renal and electrolyte parameters can reduce the adverse drug reaction and toxicity with Amphotericin B. Along with this improved strategies for the management of infusion related adverse events are required.

\section{Limitation of Study}

Renal tolerance of the various forms of Amphotericin B is not evaluated in the study since Amphotericin B lipid complex and lyophilised Amphotericin B has been withhold following the severe adverse drug reaction after the first dose. So the renal tolerance of various forms of Amphotericin B was not being evaluated.

\section{Funding None.}

\section{Declarations}

Conflict of interest The authors declare that they have no conflict of interest.

Ethical Approval The study was approved by the institutional ethics committee.

\section{References}

1. Adler-Moore JP, Proffitt RT (2002) Am Bisome: liposomal formulation, structure, mechanism of action and preclinical experience. J Antimicrob Chemother 49(S1):21-30

2. Roden MM, Nelson LD, Knudsen TA, Jarosinski PF, Starling JM, Shiflett SE, et al. (2003) Triad of acute ionrelated reactions associated with liposomal amphotericin B: analysis of clinical and epidemiological characteristics. Clin. Infect Dis. May;36(10):1213-20. [PubMed] [Google Scholar] 
3. Barcia JP (1998) Hyperkalemia associated with rapid infusion of conventional and lipid complex formulations of amphotericin B. Pharmacotherapy 18:874-876

4. Groot OA, Trofl RJ, Girbes AR, Swart NL, Beishuizen A (2008) Acute refractory hyperkalaemia and fatal cardiac arrest related to administration of liposomal amphotericin B. Neth J Med 66:433-7

5. Szebeni J, Baranyi L, Savay S, Bodo M, Morse DS, Basta M, et al. (2000) Liposome-induced pulmonary hypertension: properties and mechanism of a complement-mediated pseudoallergic reaction. Am J Physiol Heart Circ Physiol. Sep; 279(3): H1319-28. [PubMed] [Google Scholar]

6. Merhav H, Mieles L (1997) Amphotericin B lipid complex in the treatment of invasive fungal infections in the liver transplant patients. Transpl Proc 29:2670-2674

7. Wingard JR (1997) Efficacy of amphotericin B lipid complex injection (ABLC) in bone marrow recipients with life-threatening systemic nycoses. Bone Marrow Trans plant 19:343-347

8. Schrey D, Andreas H. Groll, in Side Effects of Drugs Annual, 2011

9. Clements JS Jr, Peacock JE Jr (1990) Amphotericin B reassessment of toxicity. Am J Med 88(5):22n-27n

10. Kintzel PE, Smith GH. (1992) Practical guidelines for the pulmonary aspergillosis. Antimicrobiol Agents Chemopreparing and administering amphotericin B. American therapy, 38, 518-522.J Hospital Pharmacy, 49, 1156-1164

11. Hof H (2006) A new, broad-spectrum azole antifungal: posaconazole-mechanisms of action and resistance, spectrum of activity. Mycoses 49(1):2-6. https://doi.org/10.1111/j.1439-0507. 2006.01295. X

12. U.S FDA. Noxafil instruction. 2015. https://www.accessdata.fda. gov/drugsatfdadocs/label/2014/205053s1lbl.pdf. Accessed 15Apr 2020

13. Cornely OA, Maertens J, Winston DJ, Perfect J, Ullmann AJ, Walsh TJ et al (2007) Posaconazole vs. fluconazole or itraconazole prophylaxis in patients with neutropenia. N Engl J Med 356(4):348-59. https://doi.org/10.1056/NEJMoa061094

14. Ullmann AJ, Lipton JH, Vesole DH, Chandrasekar P, LangstonA Tarantolo SR et al (2007) Posaconazole or fluconazole for prophylaxis in severe graft-versus-host disease. N Engl J Med 356(4):335-47. https://doi.org/10.1056/NEJMoa061098

Publisher's Note Springer Nature remains neutral with regard to jurisdictional claims in published maps and institutional affiliations. 